\title{
Study Design of PROCEDURE Study. A Randomized Comparison of the Dose-Dependent Effects of Pitavastatin in Patients with Abdominal Aortic Aneurysm with Massive Aortic Atheroma: Prevention of Cholesterol Embolization during Endovascular and Open Aneurysm Repair with Pitavastatin (PROCEDURE) Study
}

\author{
Katsuyuki Hoshina, MD, PhD, ${ }^{1}$ Masaru Nemoto, MD,${ }^{1}$ Takuya Hashimoto, MD, PhD,${ }^{2}$ Sumio Miura, MD, PhD, ${ }^{3}$ \\ Go Urabe, MD, PhD, ${ }^{4}$ Tatsu Nakazawa, MD, PhD,${ }^{4}$ Akihiro Hosaka, MD, PhD, ${ }^{1}$ Masaaki Kato, MD, PhD, ${ }^{2}$ \\ Nobukazu Ohkubo, MD, PhD, ${ }^{2}$ Takeshi Miyairi, MD, PhD, ${ }^{3}$ Hiroyuki Okamoto, MD, PhD, ${ }^{1}$ \\ Kunihiro Shigematsu, $\mathrm{MD}, \mathrm{PhD},{ }^{1}$ and Tetsuro Miyata $\mathrm{MD}, \mathrm{PhD}^{1}$
}

\begin{abstract}
Outcomes of abdominal aortic aneurysm (AAA) repair have improved in the $\mathbf{2}$ decades since the emergence of endovascular aneurysm repair (EVAR). However, EVAR is considered a contraindication for shaggy aorta because of the high risk of shower embolization. Recently, statins have been implicated in preventing embolization in patients with shaggy aorta via its pleiotropic effects, including atheroma reduction and coronary artery stabilization. We selected pitavastatin, a statin with potent effects, discovered and developed by a Japanese company because it has shown excellent pleiotropic effects on atheromatous arteries in the Japanese population. A randomized comparison study of dose-dependent effects of pitavastatin in patients with AAA with massive atheromatous aortic thrombus (PROCEDURE study) has begun. PROCEDURE has an enrollment goal of up to 80 patients with AAA with massive aortic atheroma (excluding intrasac atheroma), randomly allocated into 2 groups receiving pitavastatin at a dose of 1 or $4 \mathrm{mg} / \mathrm{day}$. The endpoints of the PROCEDURE study include change in atheroma volume, major adverse events related to shower embolization after aneurysm repair, and lipid-lowering effects. When complete, results of the PROCEDURE study should provide objective evidence to use statins preoperatively for AAA with massive aortic atheroma.
\end{abstract}

Keywords: statin, massive atheroma, abdominal aortic aneurysms, shaggy aorta

${ }^{1}$ Division of Vascular Surgery, Department of Surgery, Graduate School of Medicine, The University of Tokyo, Tokyo, Japan

${ }^{2}$ Department of Cardiovascular Surgery, Morinomiya Hospital, Osaka, Japan

${ }^{3}$ Department of Cardiovascular surgery, Mitsui Memorial

Hospital, Tokyo, Japan

${ }^{4}$ Department of Surgery, Tokyo Metropolitan Geriatric Hospital, Tokyo, Japan

Received: July 25, 2012; Accepted: December 7, 2012

Corresponding author: Katsuyuki Hoshina, MD, PhD. Division of Vascular Surgery, Department of Surgery, Graduate School of Medicine, The University of Tokyo, 7-3-1, Hongo, Bunkyo-ku, Tokyo 113-8655, Japan

Tel: +81-3-5800-8653, Fax: +81-3-3811-6822

E-mail: traruba@gmail.com

\section{INTRODUCTION}

$\mathrm{B}$ ecause of its low-invasiveness and good initial outcomes, endovascular aneurysm repair (EVAR) has led to a paradigm shift in the treatment strategy for abdominal aortic aneurysm (AAA). In Japan, the general consensus for AAA treatment is open surgery as the firstline treatment, and EVAR, for "high-risk" patients.1) Although open surgery is strongly recommended in patients with anatomically difficult AAA for EVAR or in those with an allergy to contrast-agents, various techniques 
including $\mathrm{CO}_{2}$ angiography or intravascular ultrasonography, and the "snorkel" technique have expanded the indications for performing EVAR, which has led to an increased use of EVAR recently. ${ }^{2-4)}$

Shaggy aorta is known to cause fatal perioperative complications; therefore, open surgery is thought to be preferable in such cases. ${ }^{5,6)}$ Our previous report also revealed that outcomes of EVAR for AAA with massive aortic atheroma were worse than those of open surgery. ${ }^{7)}$ To protect against intraoperative shower embolization, "neoadjuvant" drugs, which may reduce or stabilize the atheroma have been sought.

Several recent reports have revealed the pleiotropic effects of statins, including plaque regression and atherosclerotic coronary artery stabilization..$^{8-10)}$ However, only a few reports have shown the effect of statins on the atherosclerotic aorta. Corti and Yonemura reported a regression of thoracic aortic plaque, as determined by MRI, after lipid-lowering therapy with strong statins. ${ }^{11,12)}$ However, the plaque examined in the paper was not "massive" in volume or "shaggy," and its contribution to patients' outcomes was not reported. Recent cohort studies reported clinical benefits of statins in improving the outcomes of EVAR as well as open surgery. ${ }^{13,14)}$

For this study, we selected pitavastatin, which was originally discovered and developed by a Japanese company. ${ }^{15,16)}$ The appropriate dosage and incidence of adverse reactions have been thoroughly examined in the Japanese population. ${ }^{17-20)}$ This drug has been reported to inhibit the proliferation of smooth muscle cells derived from human coronary arteries more strongly than other statins, even at low concentrations. ${ }^{21)}$ Therefore, we selected pitavastatin, to examine its dose-dependent effects on the atherosclerotic aorta for study subjects. The purpose of this study is to evaluate the effect of pitavastatin on massive aortic atheroma, including regression of atheroma volume, complications after aneurysm repair, and lipid levels.

\section{Preliminary Data}

Thoracic aortas of 22 patients with extensive atheromas were examined using contrast-enhanced computed tomography (CECT) from the ostium of the left subclavian artery to the ostium of the proximal renal artery. Extensive thoracic atheroma was defined as (1) thickness $>5 \mathrm{~mm}$, (2) circumference of the thoracic aorta involved $>50 \%$, and (3) length $>30 \mathrm{~mm}$. Three trained examiners manually measured the integrated all atheromatous areas $\left(\mathrm{cm}^{2}\right)$. The integrated atheroma areas pre- and poststatin administration and the atheroma reduction ratio (ARR) were evaluated. The atheroma areas decreased after the administration of statins, and the ARR was found to be significant $(P<0.01)$. Of the 8 patients who showed an ARR $>10 \%, 6$ had been administered strong statins that are considered to have high lipid-lowering effects. No complications associated with extensive atheroma were observed during the follow-up period (771 \pm 897 days). This pilot study indicated that statins can rapidly reduce extensive thoracic atheromas, as well as lower lipid concentrations. (In press, Nemoto, et al.)

\section{Patients And Methods}

\section{Trial Design}

This clinical trial is designed to explore the dosedependent effect of pitavastatin administration at $1 \mathrm{mg}$ vs. $4 \mathrm{mg} /$ day on massive aortic atheroma. The study endpoints include regression of the atheroma volume, postoperative complications related to shower embolization, and lipid lowering effect.

This trial was registered in the University Hospital Medical Information Network Clinical Trials Registry (UMIN-CTR) and accepted by the International Committee of Medical Journal Editors (ICMJE) (No. UMIN000008207).

\section{Inclusion Criteria}

Patients are those with AAA and/or iliac artery aneurysm accompanied with massive aortic atheroma (excluding intrasac atheroma) scheduled to undergo aneurysm repair. The definition of "massive" aortic atheroma differs with aortic sites. Atheroma from the left subclavian artery to the upper renal artery has to meet the following criteria: (1) thickness $\geq 5 \mathrm{~mm}$, (2) circumference of the infrarenal aortic neck $\geq 50 \%$, and (3) length $\geq 30 \mathrm{~mm}$ (axial section). Atheroma at the aneurysmal neck, defined as an infrarenal aorta with normal diameter, has to meet the following criteria: (1) thickness $\geq 5 \mathrm{~mm}$, (2) circumference of the infrarenal aortic neck $\geq 75 \%$, and (3) length $\geq 5 \mathrm{~mm}$ (axial section). ${ }^{7)}$ All patients are required to satisfy the criteria of dyslipidemia as specified in Table 1.

\section{Exclusion Criteria}

Exclusion criteria include continuous prescription of statins (a washout period of at least 2 weeks is necessary), chronic kidney disease (CKD; stage 4 and 5), 
Table I Study design of the PROCEDURE study

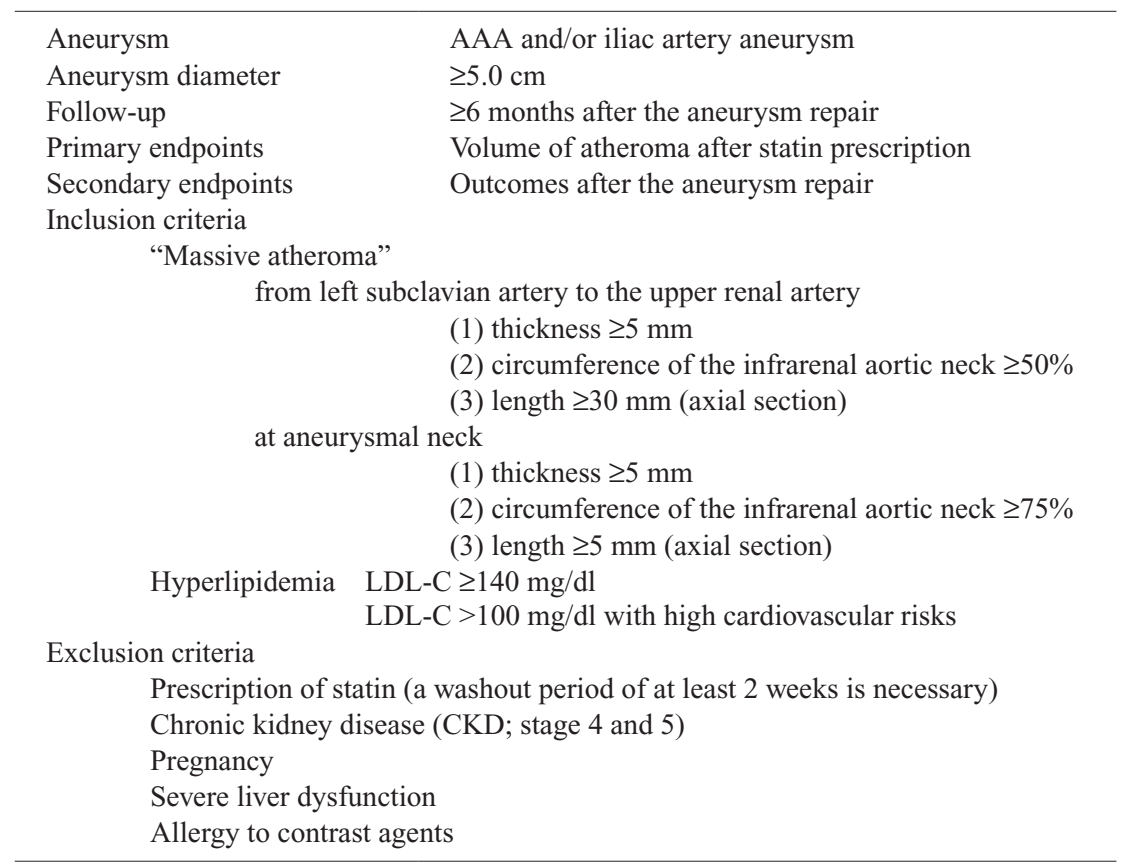

pregnancy, severe liver dysfunction, and allergy to contrast agents shown in Table $\mathbf{1}$.

\section{Study Outline}

The flow chart of the study design is shown in Fig. 1. After CECT, participants are randomly allocated to 2 groups of pitavastatin administration: $1 \mathrm{mg} /$ day or $4 \mathrm{mg} /$ day, administered for 1 month \pm 1 week, followed by reexamination of the lipid level as well as evaluation of the atheroma volume via CECT. Thereafter, EVAR or open surgery is selected, depending on patients' risks and aneurysm anatomy.

Following aneurysm repair, each group will receive pitavastatin at their earlier dosage for $6 \pm 1$ month, the final time point at which atheroma volume had changed on the CECT, adverse events related to shower embolization, ${ }^{7}$ and effect on lipid levels had been evaluated.

\section{Randomization}

Enrollment and random allocation will be performed by central registration of the University Hospital Clinical Trial Alliance Clinical Research Supporting System (UHCT ACReSS) of the University of Tokyo. Managers of each institute will enroll participants after examining their eligibility and obtaining informed consent. Patients are allocated to 2 groups via stratified block randomization, following which, the arm allocation will be notified within $24 \mathrm{~h}$.

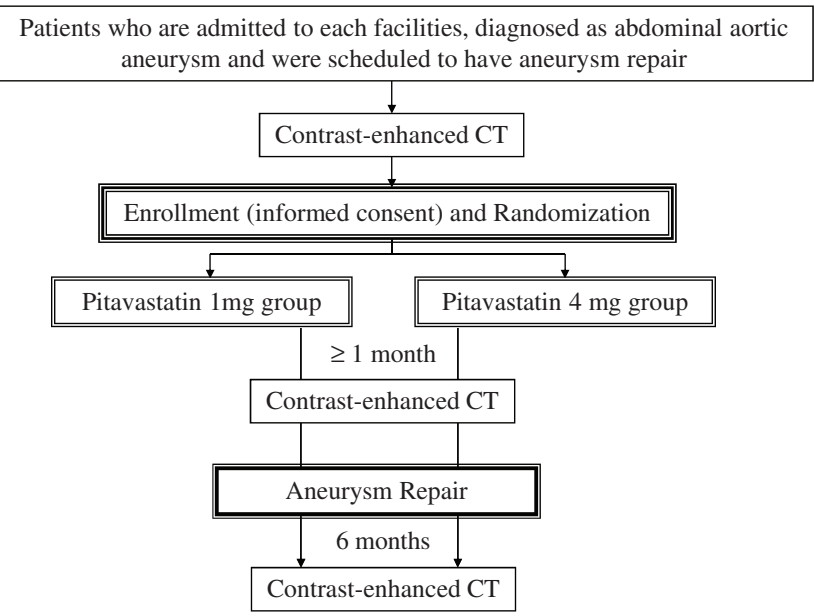

Fig. 1 Flow diagram of the study.

\section{Endpoints}

(1) The primary end point: Change in atheroma volume. At the Core Laboratory of the University of Tokyo Hospital, all CECT images were collected by a trained examiner who was blinded to the original data. Two trained examiners manually measured the integrated atheromatous area of each image using the free software program Osirix $^{\circledR}$ 3.9-DICOM viewer for Mac OS X. Atheroma volume was evaluated as the integrated area of the atheroma in each slice; the slice thickness ranged from 3 to $5 \mathrm{~mm}$. The ARR was calculated by subtracting the area $\left(\mathrm{cm}^{2}\right)$ after pitavastatin administration from the 
pre-administration area, and dividing the difference by the pre-administration area $\left(\mathrm{cm}^{2}\right)$.

(2) The secondary endpoint: Adverse event related to shower embolization and the lipid lowering effect.

Postoperative adverse events are based on the complication grade modified from the grading of the reporting standards of the Ad Hoc Committee for Standardized Reporting Practices in Vascular Surgery of the Society for Vascular Surgery/American Association for Vascular Surgery. ${ }^{7,22)}$ Grades 2 and 3 are considered to be major complications. These endpoints will be analyzed in each subgroup of EVAR and open surgery. The levels of LDL in each group will also be determined.

\section{Statistical Analysis}

Student's $t$ test is used to compare the atheroma volume change, the rate and variation of major adverse events, and the lipid-lowering rate for the duration of the study in the 2 groups.

\section{Study Organization and Study Period}

Four institutes, The University of Tokyo Hospital (Tokyo, Japan), Morinomiya Hospital (Osaka, Japan), Mitsui Memorial Hospital (Tokyo Japan), and Tokyo Metropolitan Geriatric Hospital (Tokyo Japan), are participating in the study, as of May 2, 2012. The "Aortic Atheroma Management Workshop" was set by these institutes in December 2010, and new entry of other facilities should be permitted via the Workshop. The registration period was set until March 31, 2014 initially, however, the study period should be extended under the approval of the Workshop and the IRB.

\section{Ethical Consideration}

In accordance with the Declaration of Helsinki, the ethical committee of each institute are obtaining written informed consent from all participants after explaining the complete trial.

\section{Discussion}

Pitavastatin (trade name: Livalo) was discovered in Japan by Nissan Chemical Industries and developed further by Kowa Pharmaceuticals, Tokyo, Japan. ${ }^{15)}$ Like other statins, it is an inhibitor of HMG-CoA reductase, the enzyme that catalyses the first step of cholesterol synthesis, and has been revealed to have many pleiotropic effects, including plaque stabilization, anti-oxidation, and anti-inflammation. ${ }^{16)}$ Pitavastatin was considered to be more potent than other statins because it inhibits the proliferation of smooth muscle cells derived from human coronary arteries more strongly than atorvastatin, simvastatin, fluvastatin, rosuvastatin, or pravastatin. ${ }^{21)}$ Additionally, the incidence of adverse drug reactions when using pitavastatin was lower as compared to when using other statins. ${ }^{17-20)}$ Thus, pitavastatin was selected for our study.

A previous study that examined the outcomes of aneurysm repair in AAA patients with massive aneurysm neck thrombus revealed high adverse event rates in the Japanese population. ${ }^{7)}$ In addition, aneurysm repair for shaggy aorta is also known to have poor outcomes. $\left.{ }^{5,6}\right)$ Therefore, we have used statins preoperatively in cases of massive aortic atheroma without any definite evidence as to its benefit. We believe that preoperative administration of pitavastatin will be a powerful neoadjuvant therapy for AAA patients with massive aortic atheroma.

\section{LIMITATIONS}

The study population includes patients who are admitted to institutes participating in our study; they are located in Tokyo and Osaka, which may cause selection bias. Another limitation is that we do not categorize the shape and the properties of the atheroma mentioned in a previous paper. ${ }^{7)}$

In summary, the PROCEDURE study examines the dose-dependent effects of pitavastatin on massive aortic atheroma, and studies the endpoints, including change in atheroma volume, major adverse events related to shower embolization after the aneurysm repair, and lipidlowering effect. The results of this study may provide objective evidence for the routine use of pitavastatin as neoadjuvant therapy for AAA patients with shaggy aorta.

\section{Disclosure Statement}

The authors declared no potential conflicts of interest with respect to the research, authorship, and/or publication on this article. The authors received no financial support for the research, authorship, and/or publication of this article.

\section{REFERENCES}

1) Obitsu $Y$, Ishimaru $S$, Shigematsu $H$. The education system to master endovascular aortic repair in Japanthe Japanese Committee for Stentgraft Management. Eur J Vasc Endovasc Surg 2010; 39 Suppl 1:S5-9. 
2) Hoshina K, Kato M, Miyahara T, et al. A retrospective study of intravascular ultrasound use in patients undergoing endovascular aneurysm repair: its usefulness and a description of the procedure. Eur J Vasc Endovasc Surg 2010; 40: 559-63.

3) Morito H, Hoshina K, Hosaka A, et al. Endovascular surgery for inflammatory abdominal aortic aneurysm with contrast allergy- Usefulness of carbon dioxide angiography and intravascular ultrasound: A case report. Ann Vasc Dis 2012; 5: 104-8.

4) Moulakakis KG, Mylonas SN, Avgerinos E, et al. The chimney graft technique for preserving visceral vessels during endovascular treatment of aortic pathologies. J Vasc Surg 2012; 55: 1497-503.

5) Hollier LH, Kazmier FJ, Ochsner J, et al. "Shaggy" aorta syndrome with atheromatous embolization to visceral vessels. Ann Vasc Surg 1991; 5: 439-44.

6) Zempo N, Sakano H, Ikenaga S, et al. Fatal diffuse atheromatous embolization following endovascular grafting for an abdominal aortic aneurysm: report of a case. Surg Today 2001; 31: 269-73.

7) Hoshina K, Hosaka A, Takayama T, et al. Outcomes after open surgery and endovascular aneurysm repair for abdominal aortic aneurysm in patients with massive neck atheroma. Eur J Vasc Endovasc Surg 2012; 43: 257-61.

8) Nissen SE, Tuzcu EM, Schoenhagen P, et al. Effect of intensive compared with moderate lipid-lowering therapy on progression of coronary atherosclerosis: a randomized controlled trial. JAMA 2004; 291: 1071-80.

9) Okazaki S, Yokoyama T, Miyauchi K, et al. Early statin treatment in patients with acute coronary syndrome: demonstration of the beneficial effect on atherosclerotic lesions by serial volumetric intravascular ultrasound analysis during half a year after coronary event: the ESTABLISH Study. Circulation 2004; 110: 1061-8.

10) Toi T, Taguchi I, Yoneda S, et al. Early effect of lipid-lowering therapy with pitavastatin on regression of coronary atherosclerotic plaque. Comparison with atorvastatin. Circ J 2009; 73: 1466-72.

11) Corti R, Fuster V, Fayad ZA, et al. Lipid lowering by simvastatin induces regression of human atherosclerotic lesions: two years' follow-up by high-resolution noninvasive magnetic resonance imaging. Circulation 2002; 106: 2884-7.

12) Yonemura A, Momiyama Y, Fayad ZA, et al. Effect of lipid-lowering therapy with atorvastatin on atherosclerotic aortic plaques detected by noninvasive magnetic resonance imaging. J Am Coll Cardiol 2005; 45: 733-42.

13) McNally MM, Agle SC, Parker FM, et al. Preoperative statin therapy is associated with improved outcomes and resource utilization in patients undergoing aortic aneurysm repair. J Vasc Surg 2010; 51: 1390-6.

14) Dawson JA, Choke E, Loftus IM, et al. A randomised placebo-controlled double-blind trial to evaluate lipidlowering pharmacotherapy on proteolysis and inflammation in abdominal aortic aneurysms. Eur J Vasc Endovasc Surg 2011; 41: 28-35.

15) Mukhtar RY, Reid J, Reckless JP. Pitavastatin. Int J Clin Pract 2005; 59: 239-52.

16) Saito Y. Critical appraisal of the role of pitavastatin in treating dyslipidemias and achieving lipid goals. Vasc Health Risk Manag 2009; 5: 921-36.

17) Kurihara Y, Douzono T, Kawakita K, et al. A largescale, prospective post-marketing surveillance of pitavastatin (LIVALO ${ }^{\circledR}$ Tablet) - drug use investigation. [in Japanese] Jpn Pharmacol Ther 2007; 35: 9-40.

18) Komano N, Masaki M, Kawai H, et al. The safety and efficacy in post-marketing surveys of atorvastatin. [in Japanese] Prog Med 2005; 25: 131-42.

19) Yoshida S. Crestor: Safety and efficacy in clinical experience investigation. [in Japanese] Prog Med 2007; 27: 1159-89.

20) Kurihara Y, Douzono T, Kawakita K, et al. A largescale, long-term, prospective post-marketing surveillance of pitavastatin (LIVALO ${ }^{\circledR}$ Tablet) - LIVALO Effectiveness and Safety (LIVES) Study. Jpn Pharmacol Ther 2008; 36: 709-31.

21) Nakano K, Egashira K. Pitavastatin has most potent pro-healing effects on endothelial cells and inhibitory effects on proliferation of vascular smooth muscle cells-a potential treatment strategy for drug-eluting stents. The 41st Annual Scientific Meeting of the Japan Atherosclerosis Society (July, 2009) general presentation No. 21.

22) Chaikof EL, Blankensteijn JD, Harris PL, et al. Reporting standards for endovascular aortic aneurysm repair. J Vasc Surg 2002; 35: 1048-60. 